\title{
Perfil dos idosos em situação de violência atendidos em serviço de emergência em Recife-PE
}

\author{
Profileof ddalyinvidenesitutionassited th anenergeny servicein PeifelE
}

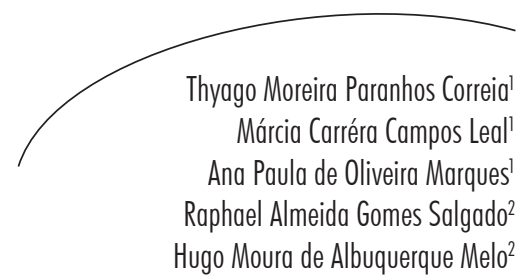

\section{Resumo}

Objetivo: Determinar o perfil da violência sofrida pelo idoso atendido em serviço de emergência do Hospital da Restauração (HR), em Recife-PE. Metodologia: Estudo epidemiológico, descritivo, quantitativo de corte transversal. Foram analisados todos os prontuários médicos referentes aos atendimentos em cirurgia geral, ortopedia e clínica médica realizados na emergência de adultos do HR entre $1^{\circ}$ de janeiro e 31 de dezembro de 2007, disponibilizados pelo Serviço de Arquivo Médico, totalizando 72.232 prontuários. Resultados: Do total de prontuários analisados, foram registrados 79 casos de violência contra o idoso. Destes, 63,3\% do sexo masculino. A faixa etária predominante foi compreendida entre $60-70$ anos (72,2\%). Em 81,1\% dos prontuários, não houve registro sobre vínculo empregatício ou situação previdenciária do agredido. Em relação ao local de residência da vítima, a maioria (46,8\%) residia em Recife-PE. Quanto ao levantamento de dados sobre o agressor, quando este foi citado (3,7\%), todos pertenciam ao sexo masculino, sendo notificada a relação com a vítima em um único caso (filho). O tipo de violência mais frequente foi a física (97,5\%). Quanto à natureza da lesão, a mais comum foi a contusão $(27,8 \%)$. Os sítios de lesão mais acometidos foram a face $(35,4 \%)$, crânio $(31,6 \%)$ e extremidades $(22,8 \%)$. Em relação ao instrumento, os contundentes foram os mais utilizados $(29,1 \%)$, seguido dos perfuro-cortantes $(19 \%)$. O mês de janeiro prevaleceu com o maior número de atendimentos $(21,6 \%)$ e os casos de violência ocorreram principalmente na sexta-feira (16,1\%), quarta-feira (17,7\%), sábado $(17,7 \%)$ e domingo (17,7\%). Conclusão: O idoso vítima de violência foi principalmente homem, com faixa etária de 60-70 anos. O tipo de violência mais frequente foi a física, especialmente contusão em face ou crânio, ocorrendo mais no fim de semana.

1 Centro de Ciências da Saúde, Departamento de Medicina Social. Universidade Federal de Pernambuco. Recife, PE, Brasil.

2 Centro de Ciências da Saúde, Departamento de Medicina Clínica. Universidade Federal de Pernambuco. Recife, PE, Brasil.

Correspondência / Correspondence

Márcia Carréra Campos Leal

E-mail: marciacarrera@hotmail.com

Palavras-chave: Agressão. Idoso. Violência. Hospitais de emergência. Recife, PE. 


\section{Abstract}

Objective: To determine the profile of violence suffered by elderly assisted at the emergency department. Methodology: Epidemiological, descriptive, quantitative crosssectional study. Were analyzed all medical records of care provided in General Surgery, Orthopedics and Internal Medicine in adult emergency between January 1st and December 31, 2007, provided by the Service Medical Records, totaling 72,232 records. Results: There were 79 cases of violence against elderly, of which $63.3 \%$ were male. The predominant age group was between $60-70$ years old $(72.2 \%)$. In $81.1 \%$ of the medical charts there was no records of employment. Regarding residence of the victim, the majority (46.8\%) lived in Recife city, Pernambuco state, Brazil. About data of the offender, when it was quoted $(3.7 \%)$, all of them were males, reported being the relationship with the victim in a unique case (child). The most frequently found violence was physical (97.5\%). The most common injury was contusion (27.8\%). The sites of injury most affected were face (35.4\%), head (31.6\%) and extremities (22.8\%). In relation to the instruments, blunt was used in $29.1 \%$, followed by piercing-cutting $(19 \%)$. January had the highest number of attendances (21.6\%). Violence occurred mainly on Fridays (16.1\%), Saturdays (17.7\%) and Sundays (17.7\%). Conclusion: The elderly victim of violence is primarily male, ranging 60-70 years of age. The most frequent type of violence is physical, especially in face or skull injury, occurring over the weekend.
Key words: Assault. Old. Violence. Emergency hospital. Recife, PE.

\section{INTRODUÇÃO}

O envelhecimento populacional fez parte da transição demográfica dos países desenvolvidos e hoje está em curso nos países em desenvolvimento. No Brasil, de acordo com dados do Instituto Brasileiro de Geografia e Estatística (IBGE), a partir do censo de 2010, infere-se que a população com 60 anos ou mais se situa em $10,9 \%$ dos atuais 190.732 .694 habitantes. ${ }^{1}$ De acordo com as projeções estatísticas, até o ano de 2025 , seremos a sexta maior população idosa do mundo em números absolutos, com mais de 32 milhões de idosos, os quais corresponderão a $15 \%$ da população. ${ }^{2}$

Com o incremento do número de idosos, crescem também os problemas relativos a essa parcela da população. A convivência dos idosos com indivíduos mais jovens e, muitas vezes, a dependência existente, seja financeira, social, física ou psicológica, podem gerar conflitos, a ponto de a relação entre ambos ficar insustentável, sem abertura para o diálogo e a argumentação franca. Isto ocorreria no âmbito familiar, institucional e no convívio social. ${ }^{3}$

A Organização Mundial da Saúde (OMS) ${ }^{4}$ define violência contra o idoso como um ato de acometimento ou omissão, que pode ser tanto intencional como involuntário. $\mathrm{O}$ abuso pode ser de natureza física ou psicológica, ou pode envolver maus-tratos de ordem financeira ou material. Qualquer que seja o tipo de abuso, certamente resultará em sofrimento desnecessário, lesão ou dor, perda ou violação dos direitos humanos e redução na qualidade de vida do idoso. ${ }^{5}$ Essa definição também foi detalhada pela Action on Elder Abuse, no Reino Unido, e adotada pela International Network for the Prevention of Elder Abuse (INPEA) em 2002. ${ }^{6}$

$\mathrm{Na}$ tentativa de uniformizar a classificação e estudo da violência a essa parcela da população, o relatório final do Estudo sobre Incidência Nacional de Violência, do Centro Nacional de Abusos contra Idosos de Washington, sintetizou os termos mais utilizados e assim os descreveu: abuso físico - uso de força física que pode resultar completamente em dano, dor ou prejuízo físico; abuso sexual - contato sexual não-consensual de qualquer pessoa com um idoso; abuso emocional ou psicológico - definido como inflição de angústia ou dor emocional; exploração financeira ou material - uso ilegal ou impróprio dos bens/ ativos de idosos; abandono - deserção do idoso por um indivíduo que teve custódia física ou tinha assumido responsabilidade por prover 
o seu cuidado; negligência - recusa ou fracasso em cumprir obrigações ou deveres para com um idoso; autonegligência - caracterizada como o comportamento de um idoso que ameace sua própria saúde ou segurança. A definição de autonegligência exclui a situação na qual uma pessoa mais velha mentalmente competente (que entende as consequências de suas decisões) toma uma decisão consciente e voluntária de se ocupar de atos que ameaçam sua saúde ou segurança. ${ }^{5}$

No Brasil, o artigo 4. ${ }^{\circ}$ do Estatuto do Idoso, aprovado em 2003, é explícito na proteção contra a violência - "nenhum idoso será objeto de qualquer tipo de negligência, discriminação, violência, crueldade ou opressão, e todo atentado a seus direitos, por ação ou omissão, será punido na forma da lei." Conforme o parágrafo $1^{\circ}$, "é dever de todos prevenir a ameaça ou violação aos direitos do idoso." ${ }^{\prime 8}$ A violência contra idosos se tornou um problema de saúde pública, em virtude da abrangência alarmante de suas consequências: traumas físicos, morais e psicoemocionais, que podem levar o idoso a incapacidade, dependência e até mesmo morte.

As informações sobre doenças, lesões e traumas provocados por causas violentas em idosos no Brasil ainda são pouco consistentes. Pesquisadores chegam a estimar que $70 \%$ das lesões e traumas sofridos pelos velhos não comparecem às estatísticas. No Brasil, há cerca de 93.000 idosos que se internam por ano por causa de quedas (53\%), violências e agressões $(27 \%)$ e acidentes de trânsito $(20 \%){ }^{9}$

Em Pernambuco, o número de casos de violência física reportados ao Instituto de Medicina Legal do Recife (IML), no período de 2004-2007, foi de 1.027 ocorrências contra idosos, sendo 99,1\% declaradas como agressão física. ${ }^{10}$ Das ocorrências registradas no Conselho do Idoso nesse Estado, agressão física correspondeu a 54,64\% no Agreste e a 46,11\% em Recife. As vítimas de violência no Agreste são em maioria mulheres (65,04\%), com maior proporção $(28,71 \%)$ entre 65 e 70 anos. Em Recife, as vitimas do sexo feminino correspondem a $70,75 \%$ e se concentram na faixa etária de 70 a 79 anos (42,3\%). A maioria dos agressores são filhos e filhas (48,93\% no Agreste e 62,18\% em Recife). ${ }^{11}$
Por tudo isso, esta pesquisa foi proposta almejando determinar o perfil da violência sofrida pelo idoso atendido em serviço de emergência do Estado de Pernambuco, com o intuito de propor políticas públicas para modificar essa realidade e contribuir com a diminuição do número de vítimas que geralmente sofrem, silenciosamente, até o fim de suas vidas.

\section{METODOLOGIA}

Trata-se de estudo epidemiológico, descritivo, quantitativo, de corte transversal. A população do estudo foi constituída por meio da análise de todos os prontuários médicos referentes aos atendimentos em cirurgia geral, ortopedia e clínica médica realizados na emergência de adultos do HR entre $1^{\circ}$ de janeiro e 31 de dezembro de 2007, os quais foram disponibilizados pelo Serviço de Arquivo Médico, totalizando 72.232 prontuários. Destes, foram identificados $79 \mathrm{com}$ registros de indivíduos com idade de 60 anos e mais, que sofreram violência e foram submetidos a exame clínico médico, no período determinado.

Os casos de agressão a idosos registrados em Pernambuco passam, em geral, pelo atendimento clínico em alguma das emergências/urgências do Estado. Pensando nesse fluxo, escolheu-se como local de pesquisa uma das maiores unidades públicas de atendimento à saúde do Nordeste, o Hospital da Restauração (HR), em Recife-PE.

O HR é um hospital de emergência, com uma média mensal de 800 internações, 700 cirurgias, 12.300 atendimentos ambulatoriais e 12.000 emergenciais. Tem um gasto anual médio de 13 milhões de reais, excluindo a folha de pagamento dos servidores. Seu quadro de pessoal é constituído por mais de dois mil funcionários, entre nível superior, médio e elementar. A clientela atendida é universalizada e gratuita, muito variada, e não conhecida em detalhes. Estima-se que sejam atendidos indivíduos de todas as classes sociais, provenientes das mais diversas regiões do Estado. ${ }^{12}$

Os dados foram coletados por meio da utilização de um formulário elaborado para a 
pesquisa, que foi preenchido de acordo com as informações contidas nos prontuários médicos obtidos no arquivo do hospital. O formulário constou de 15 questões mistas (fechadas e abertas) que contemplaram as seguintes variáveis: 1) tipo de violência - caracterizada como física, psicológica, sexual, abandono, negligência, abuso financeiro e econômico, autonegligência ou outro; 2) natureza da lesão - sem lesão, fratura, entorse/luxação, corte, perfuração, laceração, contusão, queimadura, traumatismo, politraumatismo, amputação ou outro; 3) local da lesão - crânio, face, pescoço, coluna vertebral, tórax, abdome ou extremidades; 4) instrumento utilizado - objeto utilizado; 5) encaminhamento após atendimento - tipo de encaminhamento; 6) fatores associados: (a) sexo - masculino ou feminino; (b) idade - definida em anos completos; (c) estado civil - solteiro, casado, união consensual, divorciado/separado, viúvo ou outro; (d) ocupação - consistirá na ocupação informada no prontuário; (e) município de residência consistirá no município em que a vítima residia quando foi submetida ao exame clínico no HR; (f) bairro de residência - consistirá no bairro em que a vítima residia quando foi submetida ao exame clínico; (g) dia da semana - consistirá no dia da semana em que a violência foi cometida; (h) local da ocorrência - consistirá no local em que a vítima foi submetida à agressão;(i) sexo do agressor - caracterizado como "masculino" ou "feminino"; (j) relação estabelecida com o idoso - filho(a), neto(a), companheiro(a), irmão(a), genro/nora, outro familiar, vizinho(a), cuidador(a), desconhecido(a) ou outro.

A coleta teve início após a aprovação pelo Comitê de Ética em Pesquisa do Centro de Ciências da Saúde da Universidade Federal de Pernambuco - protocolo: $\mathrm{n}^{\circ}$ 222/08 (registro do SISNEP FR - 208031 CAAE - 0216.0.172.000-08).

\section{RESULTADOS E DISCUSSÃO}

Foram encontrados 79 casos de violência contra o idoso no período do estudo. Segundo as variáveis sociodemográficas, o perfil do idoso vitimado foi de homens $(63 \%)$ na faixa etária de 60 a 70 anos
$(72,2 \%)$. Observa-se uma diferença na prevalência dos casos entre os sexos dessa amostra, ao se comparar com aqueles notificados no Brasil em 2006-2007, nos quais houve predomínio do sexo feminino $(65 \%),{ }^{13}$ fato também observado em pesquisa que descreve a situação de pessoas idosas em Moçambique, na África. ${ }^{14}$ Deve-se considerar, contudo, as características do presente estudo que, por sua metodologia e tipo de amostra, atende somente à validade interna.

As informações sobre o estado civil e ocupação não constavam na maioria dos prontuários (97,5\% e 81,1\%, respectivamente). O maior percentual dos pacientes em questão residia em Recife (46,8\%).

Não houve menção de dados a respeito do agressor na maioria dos prontuários estudados (97,3\%). Quando citado, o mesmo pertencia em todos os casos ao sexo masculino (3,7\%). Vale ainda salientar que a relação estabelecida vítimaagressor foi notificada em apenas um caso (filho). Então, devido ao inadequado preenchimento dos prontuários, não foi possível caracterizar o perfil do agressor que, conforme a literatura, em grande parte dos casos (80\%), é um familiar ou conhecido próximo da vítima. ${ }^{15}$

Outros estudos ${ }^{16,17}$ revelam pontos de convergência a este respeito e indicam que: os principais agressores são os filhos homens, noras, genros e cônjuges; há forte associação com o uso de álcool e drogas; há relação de dependência financeira entre pais e filhos; há história de violência na família e sofrimento mental e psiquiátrico, entre outros. Devido aos vínculos de afeto e dependência entre idoso e o agressor familiar, aumenta-se a dificuldade em revelar os abusos sofridos, em função do medo de retaliação ou vergonha do ocorrido, fazendo com que grande parte dos maus-tratos permaneça silenciada. ${ }^{16,17}$

Os gráficos 1 a 4 apresentam a distribuição dos idosos, segundo o tipo de violência sofrida. Evidenciou-se predominância de agressão física (97,5\%); algumas ocorrências de violência do tipo sexual e psicológica também foram encontradas (3,7\% e 2,5\%, respectivamente). Esses dados 
mostram ordem inversa ao notificado no Brasil em 2006-2007, em que prevaleceu a violência do tipo psicológica/moral (55\%), sucedida pela física $(27 \%)$ e pela sexual $(4 \%){ }^{13}$

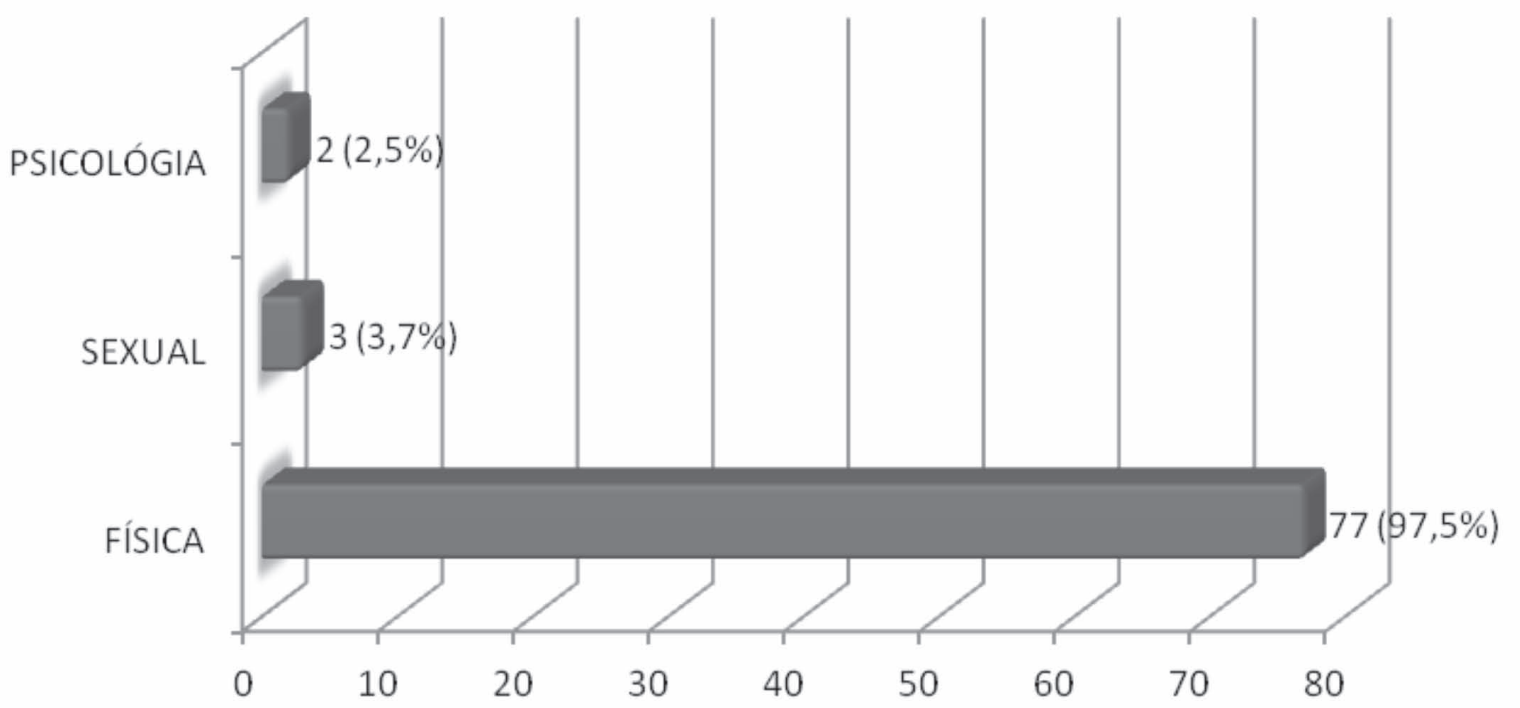

Gráfico 1 - Distribuição dos idosos vítimas de violência atendidos em Serviço de Emergência, segundo tipo de violência. Recife, 2010.

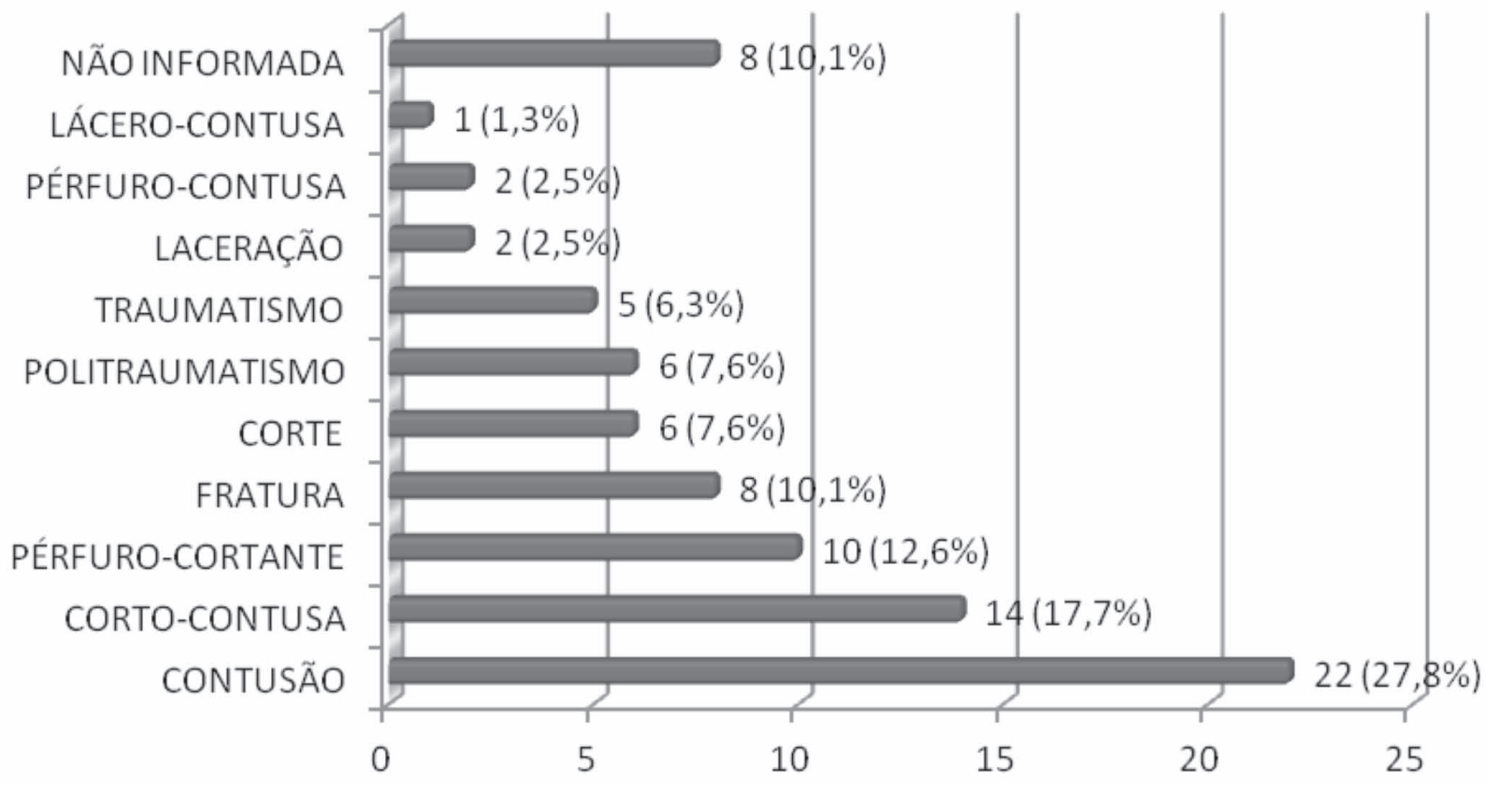

Gráfico 2 - Distribuição dos idosos vítimas de violência atendidos em Serviço de Emergência, segundo natureza da lesão. Recife, 2010. 


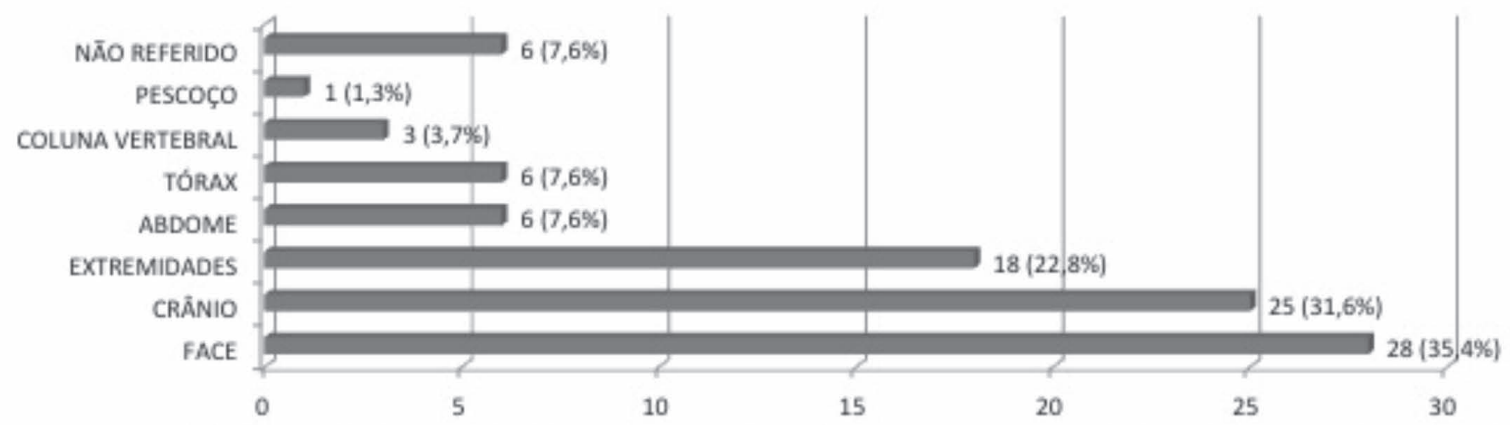

Gráfico 3 - Distribuição dos idosos vítimas de violência atendidos em Serviço de Emergência, segundo local da lesão. Recife, 2010.

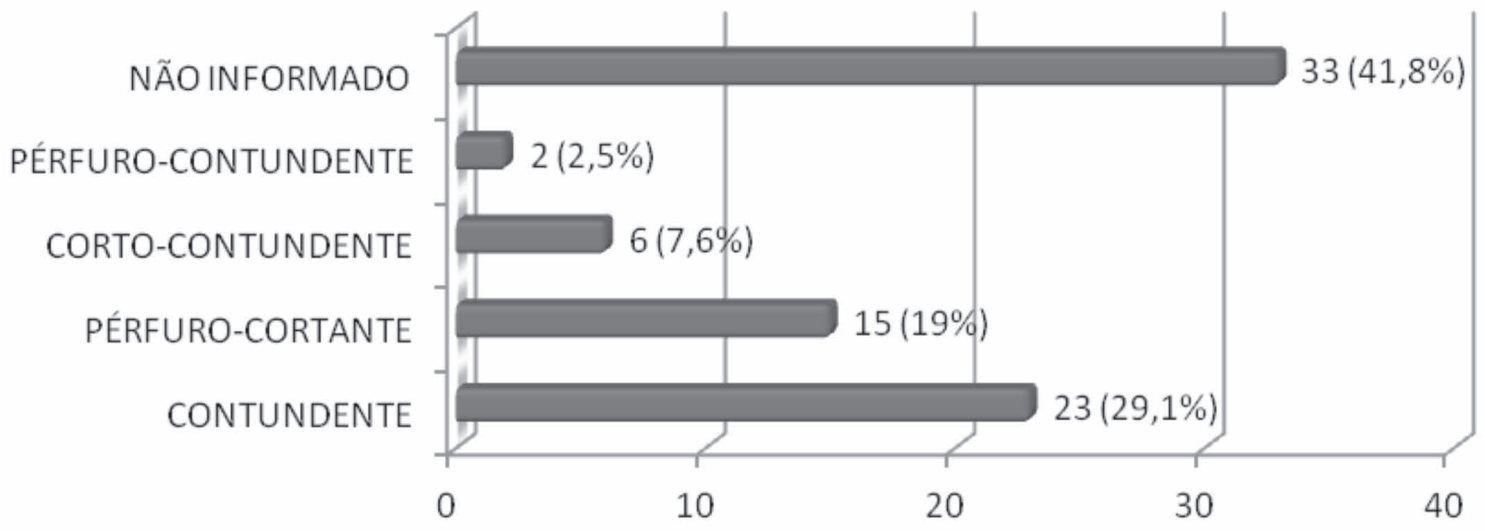

Gráfico 4 - Distribuição dos idosos vítimas de violência atendidos em Serviço de Emergência, segundo instrumento utilizado. Recife, 2010.

Em estudos realizados nos países desenvolvidos, observa-se umperfil relativamente diferente do Brasil quanto à prevalência do tipo de violência direcionada aos idosos. Nos Estados Unidos(EUA) a autonegligência é a condição mais comum entre os casos relatados, e as estimativas da prevalência de abuso de idosos na comunidade ficam entre 2-10\%. ${ }^{18}$ Ainda entre os norte-americanos, o Centro Nacional sobre Abuso em Idosos (NCEA) pesquisou todas as agências do Serviço de Proteção aos Adultos (APS) dos EUA em 2004. ${ }^{19}$ Em pouco mais de
250.000 relatórios envolvendo adultos com mais de 60 anos de idade, a autonegligência foi o tipo mais comum (39\%), seguido pela negligência do cuidador $(21,5 \%)$ e exploração financeira (14\%). A violência física ficou com taxas em torno de 0,2\%, como observado no Projeto Nacional sobre Vida Social, Saúde e Envelhecimento, um estudo com representatividade nacional nos EUA, que entrevistou a comunidade de adultos mais velhos (com idade entre 57-85) sobre as experiências de maus-tratos. ${ }^{20}$ 
Quanto à natureza foram observadas, mais comumente, lesões contusas $(27,8 \%)$ e cortocontusas $(17,7 \%)$. Em relação ao local da lesão, crânio, face e extremidades foram as regiões mais atingidas (89,8\%); fato corroborado pelas notificações no Brasil em 2006-2007.13 Os instrumentos mais utilizados pelos agressores foram do tipo contundente $(29,1 \%)$, sendo relevante a ausência de descrição dos mesmos (41,8\%).

Segundo recente pesquisa sobre violência física contra a pessoa idosa em serviço médicolegal do Recife-PE, foi observado que, dos episódios causados por violência mecânica, $89,5 \%$ foram representados por lesões contusas, ${ }^{10}$ sendo este também o principal tipo de abuso registrado no presente estudo.

A respeito do mês de ocorrência da agressão, janeiro prevaleceu (21,8\%), seguido pelos meses de agosto $(14 \%)$ e junho $(11,4 \%)$. Segundo dados obtidos, os dias da semana de maior ocorrência foram quarta-feira, sábado e domingo (com $17,7 \%$, cada), seguidos pela sexta-feira $(16,1 \%)$. Em outra fonte, $35 \%$ dos casos de violência contra idosos nos exames médico-legais em Recife-PE, no período de 2004-2007, foram noticiados no sábado e no domingo. ${ }^{10}$ Outros estudos também corroboram esses dados, justificados pelo fato de muitos dos potenciais agressores consumirem bebida alcoólica nesse período. ${ }^{21-23}$

\section{REFERÊNCIAS}

1. Instituto Brasileiro de Geografia e Estatística [homepage na Internet]. Resultados do Censo 2010. [acesso em 17 out 2011]. Disponível em: <http:// www.censo2010.ibge.gov.br/resultados_do_ censo2010.php>.

2. Leal MCC, et al. Perfil de instituições asilares no município do Recife, PE, Brasil. Rev Bras Geriatr Gerontol. 2006; 9(3):39-48.

3. Florêncio MVL, Ferreira Filha MO, Sá LD. A violência contra o idoso: dimensão ética e política de uma problemática em ascensão. Revista Eletrônica de Enfermagem [serial on line] 2007 [acesso em 16 out 2011] ; 9(3):847-857.Disponível em: <http:// www.fen.ufg.br/revista/v9/n3/v9n3a23.htm>.

\section{CONCLUSÃO}

A análise dos dados denotou que o padrão do idoso vítima de violência compreendeu principalmente homens com idade entre 60-70 anos impostos à violência física por instrumentos contusos em face ou crânio. O grande número de prontuários preenchidos inadequadamente impossibilitou as seguintes caracterizações: do agressor, de um perfil mais apurado da vítima e do local de ocorrência do delito. Então, é fundamental tomar medidas que visem a informar/instruir os profissionais de saúde sobre a importância da correta inserção de dados nos formulários das unidades de saúde, que, muitas vezes por pressa, elevada demanda de pacientes ou descaso, não é realizada.

É imprescindível a realização de novos estudos sobre o tema, para melhor caracterizar a realidade da violência contra o idoso e poder intervir em sua prevenção.

\section{AGRADECIMENTOS}

Ao apoio do Fundo de Amparo a Ciência e Tecnologia do Estado de Pernambuco FACEPE. Bolsa de Iniciação Científica no período de agosto de 2009 a julho de 2010.

4. WORLD HEALTH ORGANIZATION. World Report on Violence and Health: Summary = Relatório Mundial sobre Violência e Saúde. Geneva: WHO, 2002.

5. Sanches PARA, Lebrão ML, Duarte YAO. Violência contra idosos: uma questão nova?. Saude soc. [periódicos na Internet] 2008 set[acesso em 17 out 2011]; 17(3) Disponível em: http://apsp.org.br/ saudesociedade/

6. World Health Organization. Missing voices: views of older persons on elder abuse. Geneva, World Health Organization, 2002.

7. TATARA, T. et al. The National Center on Elder Abuse. The National Elder Abuse Incidence Study Final Report. Washington, DC, 1998, 136p. 
8. BRASIL. Estatuto do idoso. Brasília: Senado Federal, Subsecretaria de Edições Técnicas, 2003.

9. Minayo MCS. Violência contra idosos: relevância para um velho problema. Cad Saúde Pública 2003; 19(3): 783-791.

10. Abath MB, Leal MCC, Melo Filho DA, Marques APO. Cad. Saúde Pública 2010; 26(9): 1797-1806.

11. Furtado, B.M.A.S.M., et al. O perfil da emergência do Hospital da Restauração: uma análise dos possíveis impactos após a municipalização dos serviços de saúde. Rev. bras. epidemiol., 2004, 7(3):279-289.

12. Faleiros, VP. Violência contra a pessoa idosa ocorrências, vítimas e agressores. Brasília: Universa, 2007. p 190

13. Brasil. Ministério da Saúde. Caracterização das vítimas de violência doméstica, sexual e outras violências interpessoais notificados no VIVA Brasil. Brasília, 2008.

14. Silva T. Violência contra a pessoa idosa: do invisível ao visível. Revista Kairós Gerontologia 2011 14(1): 65-78.

15. Brasil. Ministério da Justiça. Secretária Nacional de Direitos Humanos. Política Nacional do Idoso: Boletim. Brasília: Ministério da Justiça. 2001.
16. Collins KA. Elder maltreatment: a review. Arch Pathol Lab Med 2006; 130: 1290- 96.

17. Minayo MCS. Violência contra a pessoa idosa: o direito pelo avesso. In: Papaléu Netto, M. Tratado de gerontologia. São Paulo: Atheneu; 2007.

18. Lachs MS, Pillemer K. Elder abuse. Lancet 2004; 364:1263.

19. Teaster, PB, Dugar, TA, Mendiondo, MS, et al. The 2004 Survey of State Adult Protective Services: Abuse of Adults 60 Years of Age and Older; Prepared for The National Center on Elder Abuse 2006. [periódicos na Internet] [acesso em 20 out 2011].Disponível em:www.ncea.aoa.gov/NCEAroot/Main_Site/pdf/214-06\%20FINAL $\% 2060+$ REPORT.pdf

20. Laumann EO, Leitsch SA, Waite LJ. Elder mistreatment in the United States: prevalence estimates from a nationally representative study. J Gerontol B Psychol Sci Soc Sci 2008; 63(4): 248- 254.

21. Kleinschmidt KC. Elder abuse: a review. Ann Emerg Med 1997; 30: 463-72

22. Melo VL, Cunha JOC, Falbo Neto GH. Maustratos contra idosos no Município de Camaragibe, Pernambuco. Rev Bras Saúde Matern Infant 2006; 6 Suppl 1:S43-8.

23. Bradley M. Elder abuse. BMJ 1996; 313: 548-50. 